\title{
Serious Deterioration of Renal Function after Percutaneous Nephrolithotomy: A Case Report
}

\author{
Heng Zhang, Houyong Zhou, Bo Song, Zhansong Zhou, Longkun $\mathbf{L i}^{*}$ \\ Urology Centre, Southwest Hospital, Third Military Medical University, Chongqing, China \\ E-mail: ${ }^{*}$ lilongk@hotmail.com \\ Received November 4, 2011; revised November 29, 2011; accepted December 11, 2011
}

\begin{abstract}
A 25-year-old man presented with stones in left kidney received the left Percutaneous nephrolithotomy (PCNL) with holmium laser lithotripsy. All the stones were successfully removed, and all the results were normal in the first three months followup. However, a deterioration of renal function was confirmed two years later. No mechanical obstruction was found with ureteroscopy. The deterioration of renal function may be induced by high internal renal pelvis pressure, injury of the laser energy, potential functional obstruction, and/or ischemia-reperfusion injury.
\end{abstract}

Keywords: Percutaneous Nephrolithotomy, Renal Function, Deterioration

\section{Introduction}

Percutaneous nephrolithotomy (PCNL) is considered to be one of the standard methods for kidney stones, due to its advantages of relative high successful rate of stone clearance, little bleeding and short-time hospitalization. However, this technique is not absolutely free from complications, such as bleeding, pelvis perforation, calices renales laceration, pleural effusion, intestinal perforation, fluid leakage, urine leakage, infection and renal microvascular arteriovenous fistula [1]. So far, PCNL is still thought to be safe for renal function with optimal results, but still lack of long-term and systematic research. Here, we report a case of serious damage of kidney function after PCNL.

\section{Case Presentation}

A 25-year-old man presented with left lumbodynia and percussion pain was confirmed to be with stones in the lower calices of the left kidney by KUB + IVP. By ultrasonography, the left kidney was $164 \mathrm{~mm} \times 94 \mathrm{~mm}$ with mild hydrocephalus, $14 \mathrm{~mm}$ of renal sinus interval, and kidney stone $(12 \mathrm{~mm} \times 10 \mathrm{~mm})$ in lower calices renales, (Figure 1). A normal function in right kidney (filtration rate of $41.52 \mathrm{ml} / \mathrm{min}$ ) and a slightly decrease in left (filtration rate of $35.76 \mathrm{ml} / \mathrm{min}$ ) were revealed by ECT. The serum creatinie and urea nitrogen were normal. The patient declined any history of hypertension, diabetes, chronic nephritis or nephrotic syndrome.

The patient received the left PCNL with holmium laser lithotripsy under general anesthesia. A single percutaneous renal nephrostomy pathway was obtained under ultrasonic guidance. The calices were successfully removed by holmium laser disintegration via a $16 \mathrm{~F}$ nephrostomy sheath, with the perfusion flow of $300 \mathrm{~mL} / \mathrm{min}$ and pressure of $194 \mathrm{mmHg}$. The whole operation time (including the pre-operational catheter implant) was about one hour, and the litrotripsy time was 25 minutes. The blood loss was about $50 \mathrm{~mL}$ without any temporary postoperative complications. The nephrostomy catheter was removed one week later and double pigtail stent was removed after a month.

All the results were normal with a close followup for three months. Unfortunately, due to some private reasons, the patient himself quit the further close followup. When he came back two years later, the ultrasonography gram revealed a significant expanded left kidney $(164 \mathrm{~mm} \times$ $94 \mathrm{~mm}$ ), filled with liquid inside and little kidney parenchyma (Figure 2). No Kidney stone was found. A deterioration of renal function was confirmed in the left kidney by ECT, with normal in right kidney as a control. The filtration rate of right kidney was $47.15 \mathrm{ml} / \mathrm{min}$ and the left was $8.31 \mathrm{ml} / \mathrm{min}$. No mechanical obstruction was confirmed with ureteroscopy. After a deep communication, the patient himself chosed a close follow-up rather than further interventions. 


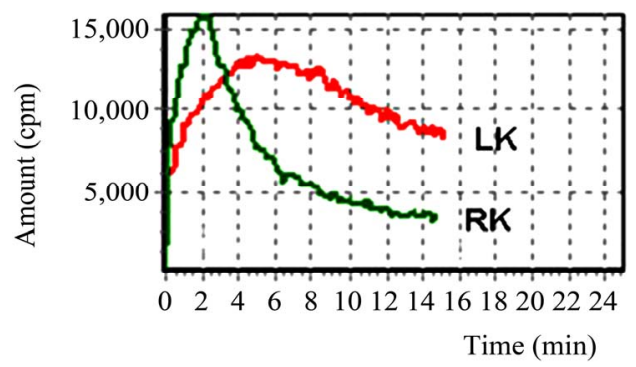

(a)

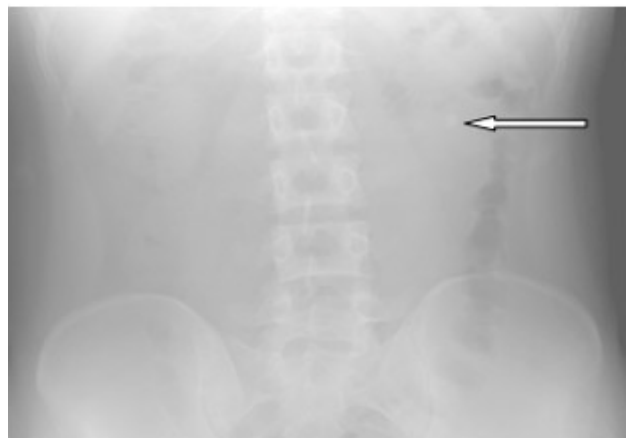

(c)

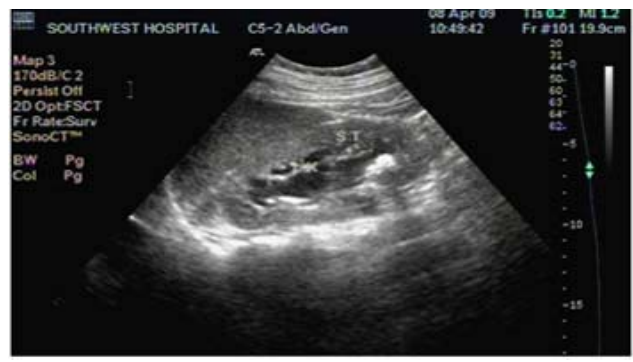

(b)

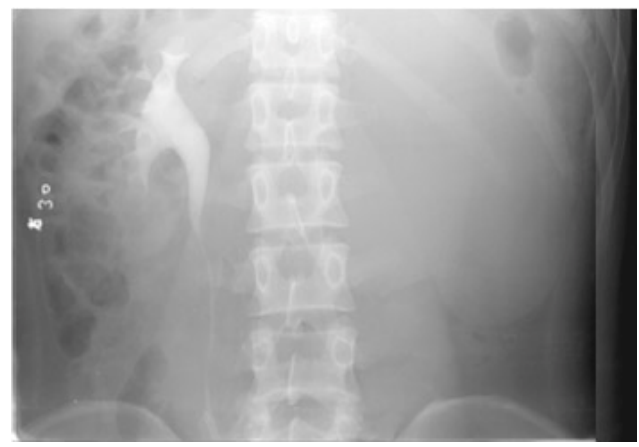

(d)

Figure 1. Pre-operational detection of renal function. (a) diuresis gram. LK: left kidney. RK: right kidney; (b) ultrasonographic gram of the left kidney. A mild hydronephrosis was confirmed. LK: left kidney; ST: stone; (c) plain film of KUB. Arrow: stone in the left kidney; (d) IVP. With mild hydronephrosis and renal function damage.

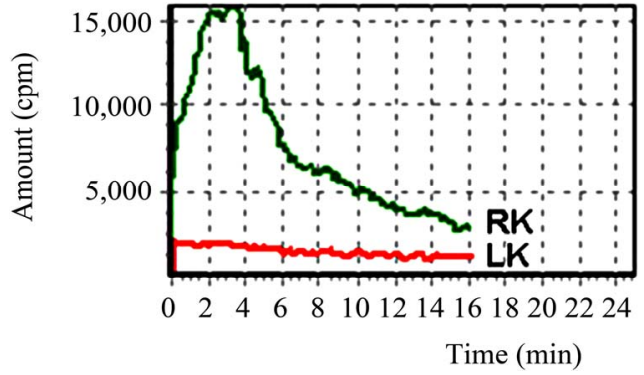

(a)

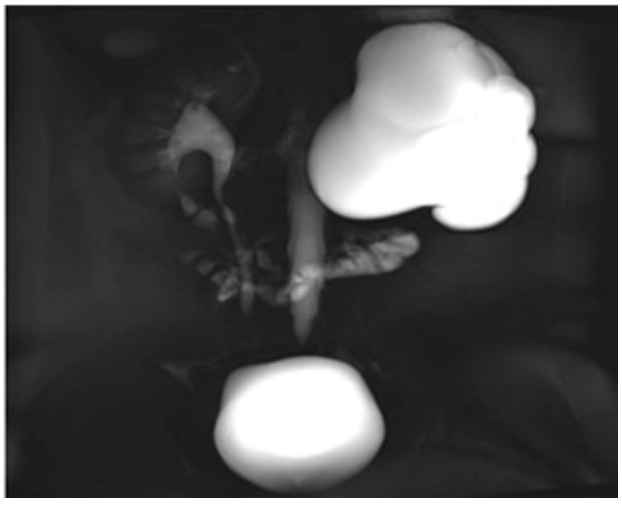

(c)

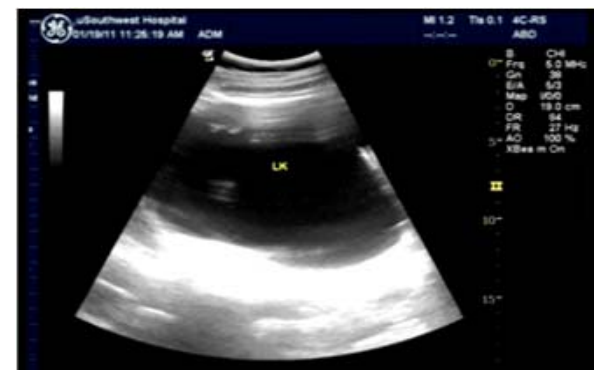

(b)

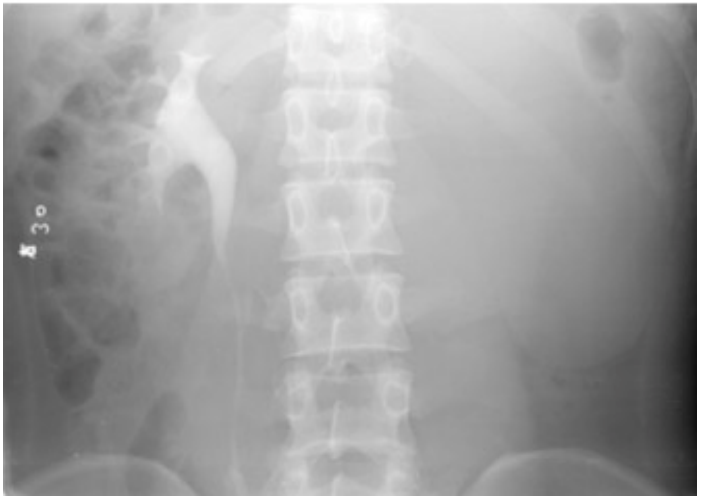

(d)

Figure 2. Post-operative detection of renal function. (a) diuresis gram. LK: left kidney. RK: right kidney; (b) ultrasonographic gram of the left kidney. A serious hydronephrosis was confirmed; (c) MRU. Significant hydronephrosis was revealed in the left kidney; (d) IVP. Almost no contrast in the left kidney area. 


\section{Disscusion}

PCNL, due to its high success rate of stone clearance and minimally invasive characteristics, has become the preferred method for renal and upper ureteral stones, especially the complex renal calculi [2]. Despite the evidence of its safety and efficacy either in adult and pediatric patients [3], or in pregnant women [4], PCNL still behaves with some complications, including the damages on the renal function. It was generally believed that the upper urinary tract obstruction, infection and interstitial renal scar caused by kidney stones, will eventually result in the damage of renal function. Segura and Liou et al. reported that PCNL could effectively remove the stones and relieve the obstruction, to maintain or even improve the renal function [5,6]. Regarding to long-term effect, there are several reports. Kuzgunbay et al. reported that most patients presenting with kidney-stome disease and renal insufficiency experience improvement or stabilization of renal function after PCNL [7]. In another study, Kuzgunbay et al. reported that patients underwent PCNL ia an adequate treatment modality even in the presence of complete staghorn calculi, comorbid diseases or previous ipsilateral renal surgery [8]. In another study carried by El-Nahas et al., it was reported that long-term functional results of PCNL were satisfactory as $91.5 \%$ of kidneys showed stable or improved GFR [9].

In this case, a serious deterioration of renal function after percutaneous nephrolithotomy was confirmed. The preoperative renal function had just mildly declined, but almost completely lost after PCNL two years later, without any evidence of secondary ureteral obstruction, chronic nephritis or nephrotic syndrome. Theoretically, there seems to be some potential mechanisms associated with the renal function deterioration, such as: 1) high internal renal pelvis pressure, due to the necessary pressure by irrigation for high qualified observation view and stone removal [10]. But when the perfusion pressure rises to $300 \mathrm{mmHg}$, the intrapelvic pressure may rise to more than $40 \mathrm{cmH}_{2} \mathrm{O}$, which may result in pyelosinus, pyelovenous, and/or pyelolymphatic backflow. 2) Injury of the laser energy. Laser could disintegrate the stone with delicacy, but could result in the risk of perforation and injury to the urothelium at the same time. 3) Potential functional obstruction. If the pelvis-uretheral junction was damaged, the ureteral peristaltic movement might be depressed, which might cause a secondary functional obstruction. 4) Ischemia-reperfusion injury. Once the renal interstitium edema gradually increased, the pressure of interstitium would exceed that of the renal arteries, resulting in the renal ischemia-reperfusion injury, stagnation of artery blood flow and formation of micro-thrombosis. Even the ischemic renal necrosis and fibrosis, which influenced by stone size, operation time, preoperative renal function, blood loss and blood pressure control and so on, can cause the damage to the renal function. Moreover, the renal function deterioration was correlated with the long-time infection that caused by kidney stone, intraoperative mucosal injury, residual stones and indwelling double-pigtail stent, which may result in the chronic kidney inflammation and fibrosis [11]. Moreover, although the patient was diagnosed without any metabolic adnormalities, he had not been deeply checked up. These would remind us to pay attention to the patients underwent PCNL.

In conclusion, PCNL is still considered as a safe and effective endoscopic technique for urinary stones. But we should pay more attention to preserve the renal function in PCNL, including to adopt small tract, reduce perfusion pressure and bleeding, shorten operation time, control preoperative and postoperative urinary tract infections, and maintain the patency of the urinary drainage.

\section{References}

[1] M. S. Michel, L. Trojan and J. J. Rassweiler, "Complications in Percutaneous Nephrolithotomy,” European Urology, Vol. 51, No. 4, 2007, pp. 899-906. doi:10.1016/j.eururo.2006.10.020

[2] R. Goel, M. Aron, P. K. Kesarwani, P. N. Dogra, A. K. Hemal and N. P. Gupta, "Percutaneous Antegrade Removal of Impacted Upper-Ureteral Calculi: Still the Treatment of Choice in Developing Countries,” Journal of Endourology, Vol. 19, No. 1, 2005, pp. 54-57. doi:10.1089/end.2005.19.54

[3] A. R. El-Nahas, A. A. Shokeir, M. R. El-Kenawy, A. M. Shoma, I. Eraky, A. M. El-Assmy, et al., "Safety and Efficacy of Supracostal Percutaneous Nephrolithotomy in Pediatric Patients,” Journal of Endourology, Vol. 180, No. 2, 2008, pp. 676-680. doi:10.1016/j.juro.2008.04.046

[4] L. Khoo, K. Anson, U. Patel, "Success and Short-Term Complication Rates of Percutaneous Nephrostomy during Pregnancy," Journal of Vascular and Interventional Radiology, Vol. 15, No.12, 2004, pp. 1469-1473.

[5] J. W. Segura, D. E. Patterson, A. J. LeRoy, H. J. Williams Jr., D. M. Barrett, R. C. Benson Jr., et al., "Percutaneous Removal of Kidney Stones: Review of 1,000 Cases,” Journal of Endourology, Vol. 134, No. 6, 1985, pp. 1077-1081.

[6] L. S. Liou and S. B. Streem, "Long-Term Renal Functional Effects of Shock Wave Lithotripsy, Percutaneous Nephrolithotomy and Combination Therapy: A Comparative Study of Patients with Solitary Kidney," Journal of Endourology, Vol. 166, No. 1, 2001, pp. 36-37. doi:10.1016/S0022-5347(05)66070-3

[7] B. Kuzgunbay, et al., "Long-Term Renal Function and Stone Recurrence after Percutaneous Nephrolithotomy in Patients with Renal Insufficiency,” Journal of Endourology, Vol. 24, No. 2, 2010, pp. 305-308. doi:10.1089/end.2009.0362 
[8] B. Kuzgunbay, et al., "Percutaneous Nephrolithotomy for Staghorn Kidney Stones in Elderly Patients," International Urology and Nephrology, Vol. 43, No. 3, 2011, pp. 639-643. doi:10.1007/s11255-010-9885-6

[9] A. R. El-Nahas, et al., "Long-Term Results of Percutaneous Nephrolithotomy for Treatment of Staghorn Stones," BJU International, Vol. 108, No. 5, 2011, pp. 750-754.

[10] J. Rehman, M. Monga, J. Landman, D. I. Lee, T. Felfela, M. C. Conradie, et al., "Characterization of Intrapelvic
Pressure during Ureteropyeloscopy with Ureteral Access Sheaths,” Urology, Vol. 61, No. 4, 2003, pp. 713-718. doi:10.1016/S0090-4295(02)02440-8

[11] R. Kukreja, M. Desai, S. H. Patel and M. R. Desai, “Nephrolithiasis Associated with Renal Insufficiency: Factors Predicting Outcome,” Journal of Endourology, Vol. 17, No. 10, 2003, pp. 875-879. doi:10.1089/089277903772036181 